\title{
Structure of an Al-Fe-Ni Decagonal Quasicrystal Studied by Cs-Corrected STEM
}

\author{
A. Yasuhara ${ }^{a *}, \mathrm{~K} . \mathrm{Yamamoto}^{b}, \mathrm{~K} \mathrm{Yubuta}^{c}$ And K. Hiraga ${ }^{c}$ \\ ${ }^{a}$ EM Application Group, EM Business Unit, JEOL Ltd., Tokyo 196-8558, Japan \\ ${ }^{b}$ Department of Physics, Nara Women's University, Nara 630-8506, Japan \\ ${ }^{c}$ Institute for Materials Research, Tohoku University, Sendai 980-8577, Japan
}

\begin{abstract}
The structure of an Al-Fe-Ni decagonal quasicrystal with two quasiperiodic planes along the periodic axis in an $\mathrm{Al}_{72} \mathrm{Ni}_{24} \mathrm{Fe}_{4}$ alloy has been examined by spherical aberration (Cs)-corrected scanning transmission electron microscopy with high-angle annular dark-field and annular bright-field techniques. The transition-metal atoms and mixed sites (MSs) of $\mathrm{Al}$ and transition-metal atoms are represented as separated bright dots in the observed highangle annular dark-field scanning transmission electron microscopy images, and consequently the arrangements of transition-metal atoms and mixed sites on the two quasiperiodic planes can be directly determined. The transitionmetal atoms are arranged on a pentagonal tiling of an edge-length of $0.76 \mathrm{~nm}$. The close examination of observed annular bright-field- and high-angle annular dark-field scanning transmission electron microscopy images indicates the existence of large decagonal columnar clusters with $3.2 \mathrm{~nm}$ diameter, and their arrangement on pentagonal, thin rhombic and squashed hexagonal tiles with an edge-length of $3.2 \mathrm{~nm}$. The arrangements of transition-metal atoms in these three tiles are placed on an ideal pentagonal tiling with an edge-length of $3.2 \mathrm{~nm}$, which is generated by the projection of a five-dimensional hyper-cubic lattice. The vertices are denoted by 5D hyper-cubic indices and then they are projected on the occupation domains in perpendicular space. The arrangement of $\mathrm{Al}$ atoms as well as transition-metal atoms and mixed sites in the large decagonal atom cluster with about $3.2 \mathrm{~nm}$ diameter is interpreted from the observed high-angle annular dark-field- and annular bright-field scanning transmission electron microscopy ABF-STEM images.
\end{abstract}

DOI: 10.12693/APhysPolA.126.637

PACS: 61.66.-f

\section{Introduction}

Recently, we have extensively re-examined the structures of Al-transition-metal (TM) decagonal quasicrystals and their crystalline approximants by Cs-corrected annular bright-field (HAADF) scanning transmission electron microscopy (STEM) HAADF-STEM, which has enough resolution to represent individual TM atoms as separated bright dots in observed images of decagonal quasicrystals. We have proposed new structure models characterized by bond orientational order (BOO) arrangements of TM atoms [1-5] instead of previous cluster-based models that have been widely accepted for a long time.

The Al-Ni-Fe decagonal quasicrystal was first found in rapidly solidified alloys [6], and then found as a stable phase in a conventionally solidified $\mathrm{Al}_{71} \mathrm{Ni}_{24} \mathrm{Fe}_{5}$ alloy [7]. This stable decagonal phase is also known to be a highlyordered one $[8,9]$.

In the present paper, we aim to produce a new interpretation about the structure of this Al-Ni-Fe decagonal quasicrystal by the full use of Cs-corrected STEM with HAADF and annular bright-field (ABF) techniques and to obtain an occupation domain for the arrangement of TM atoms in perpendicular space.

*corresponding author; e-mail: ayasuhar@jeol.co.jp

\section{Experimental procedures}

An alloy of a nominal composition of $\mathrm{Al}_{72} \mathrm{Ni}_{24} \mathrm{Fe}_{4}$ was prepared by melting high-purity $(99.99 \%)$ metals of $\mathrm{Al}$, $\mathrm{Ni}$ and $\mathrm{Fe}$ in an arc furnace under Ar atmosphere. The ingot was sealed in an evacuated quartz tube and annealed at $900^{\circ} \mathrm{C}$ for $40 \mathrm{~h} \mathrm{HAADF}$ - and ABF-STEM images were simultaneously taken with the incident beam parallel to the periodic axis by using a Cs-corrected electron microscope (JEM-ARM200F). The STEM images presented in this paper are filtered by reconstructing using Fourier diffractograms of the original images and apertures surrounding diffraction spots in the Fourier diffractograms, in order to reduce noises in the original images. The composition of the Al-Ni-Fe decagonal quasicrystal was determined by energy dispersive X-ray Spectroscopy (EDS) to be $\mathrm{Al}_{71} \mathrm{Ni}_{24} \mathrm{Fe}_{5}$, which is similar to the nominal composition.

\section{Experimental result and discussion}

Figure 1a is an ABF-STEM image of the Al-Ni-Fe decagonal quasicrystal, and all atomic columns are considered to be represented as separated dark dots along the periodic axis. In the image, one can see that small rings formed by ten dark dots are arranged with an interval about $0.66 \mathrm{~nm}$. In the arrangement of the ring contrasts, definite large clusters formed by ten small rings encircling with ten-fold rotational symmetry are observed, as indicated by circles. The large clusters are arranged on BOO 
with a bond length of $3.2 \mathrm{~nm}$. Figure $1 \mathrm{~b}$ is a HAADFSTEM image simultaneously taken with Fig. 1a. From the close examination of bright dots in Fig. 1b, one can recognize that large decagonal clusters with $3.2 \mathrm{~nm}$ diameter exist at the positions of the clusters in Fig. 1a and that they are arranged by edge-shearing and interpenetrating linkages, as indicated by the decagonal frames.
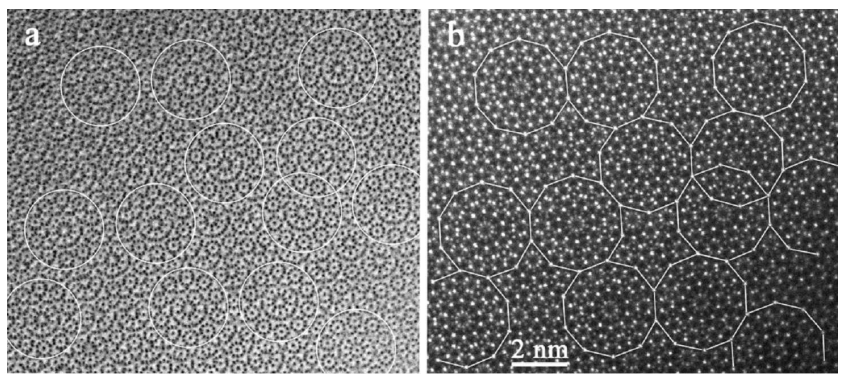

Fig. 1. ABF-STEM (a) and HAADF-STEM (b) images of the Al-Fe-Ni decagonal quasicrystal simultaneously taken with the incident beam parallel to the periodic axis. Note the large clusters, indicated by circles in (a), formed by ten small rings encircling with tenfold rotational symmetry, and a BOO arrangement of the decagonal clusters by edge-sharing and interpenetrating linkages with a bond-length of $3.2 \mathrm{~nm}$ in (b).
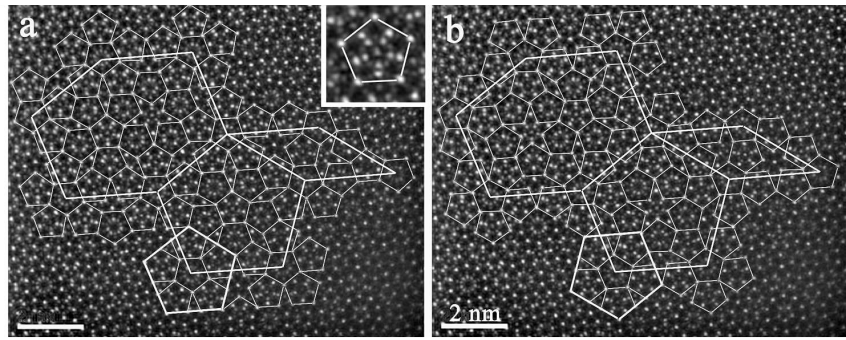

Fig. 2. Enlarged images of a part of Fig. 1b. Most of the bright dots in the image are connected by two pentagonal tilings with an edge-length of $0.76 \mathrm{~nm}$, as indicated by thin lines in (a) and (b). Note that double pentagonal arrangements of bright dots, which can be clearly seen in an enlarged image inserted in (a), are observed in upward pentagonal frames in (a) and in the downward ones in (b). Large clusters with pentagonal symmetry, indicated by thick lines, are observed at most of the vertices of the BOO tiling with an edge-length of $3.2 \mathrm{~nm}$.

Figure 2 is an enlarged HAADF-STEM image of a part of Fig. 1b. As indicated by the thin lines in Fig. 2a and b, most of the bright dots in the image can be connected by two types of pentagonal tilings with a bond-length of $0.76 \mathrm{~nm}$. In upward pentagonal frames in the pentagonal tiling of Fig. 2a and downward ones in Fig. 2b, one can recognize double pentagonal arrangements, which are composed of pairs of bright dots along the directions of fivefold rotational symmetry, as can be clearly seen in an enlarged image inserted in Fig. 2a. From the previous interpretations $[1,3,5]$, it can be concluded that all bright dots connected by lines in Fig. 2a and in Fig. 2b correspond to the TM atoms respectively on A and B quasiperiodic planes stacked along the periodic axis. Furthermore, the innermost five dots in the double pentagonal arrangements correspond to the mixed sites (MSs) in the tiling plane, and the outer five dots in these double pentagonal arrangements are the TM and/or MSs in a different plane. The unevenness of the brightness of the bright dots corresponding to MSs suggests the $\mathrm{Al} / \mathrm{TM}$ ratio being different at every MS position. On the other hand, the contrast distributions in downward pentagons in Fig. 2a and in upward ones in Fig. 2b have asymmetric arrangements of bright dots. The positions of bright dots in the asymmetric arrangements have been interpreted in the previous paper for a decagonal quasicrystal in an $\mathrm{Al}_{72} \mathrm{Co}_{8} \mathrm{Ni}_{20}$ alloy [5], but they are not mentioned in the present paper, because of their indefinite arrangements. The large clusters with pentagonal symmetry, indicated by pentagons with thick lines in Fig. 2, are observed at most of vertices of the BOO tiling with an edge-length of $3.2 \mathrm{~nm}$. Those clusters correspond to the decagonal ones in Fig. $1 b$.

From the HAADF-STEM image of Fig. 2, the arrangements of TM atoms and MSs on the A and B planes can be directly derived, as shown in Fig. 3. All the TM atoms and MSs in Fig. 3 are located at the vertices of a Penrose tiling, a part of which is inserted in Fig. 3a, with an edge-length of $0.25 \mathrm{~nm}$, and consequently the TM atoms and MSs are arranged with BOO. The pentagonal arrangements of MSs in pentagonal frames with definite directions can be seen in the $\mathrm{B}$ plane of the structure of $\mathrm{W}-(\mathrm{AlCoNi})[1,10]$ and $\tau^{2}-\mathrm{Al}_{3} \mathrm{Co}[2]$ crystalline phases, which are considered to be important approximants for the interpretation of Al-TM decagonal quasicrystals and in quasiperiodic planes of several types of Al-Co-Ni decagonal quasicrystals $[1,4,5]$.

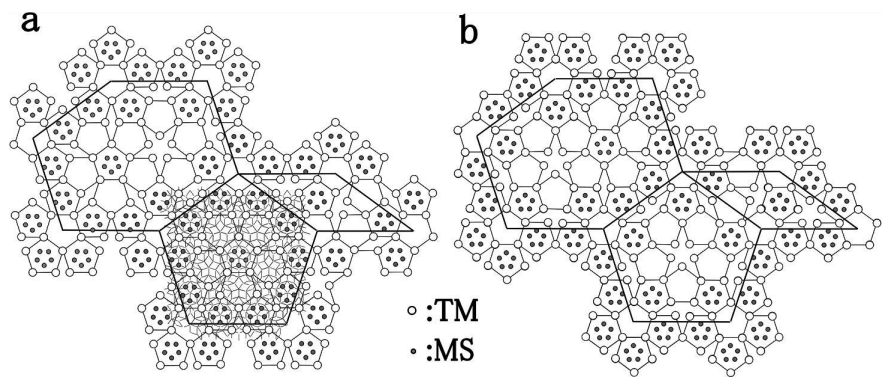

Fig. 3. Arrangements of TM atoms and MSs in the A (a) and B (b) quasiperiodic planes stacking along the periodic axis, derived from the arrangements of bright dots in Fig. 2. Note that the pentagonal arrangements of MSs are located at upward pentagonal frames in (a) and downward ones in (b). All the TM atoms and MSs are located at the vertices of a Penrose tiling, a part of which is inserted in (a), with an edge-length of $0.25 \mathrm{~nm}$.

The TM atoms in squashed hexagonal, thin rhombic, and pentagonal frames with an edge-length of $3.2 \mathrm{~nm}$ are placed in an ideal pentagonal tiling of Fig. 4a, which 

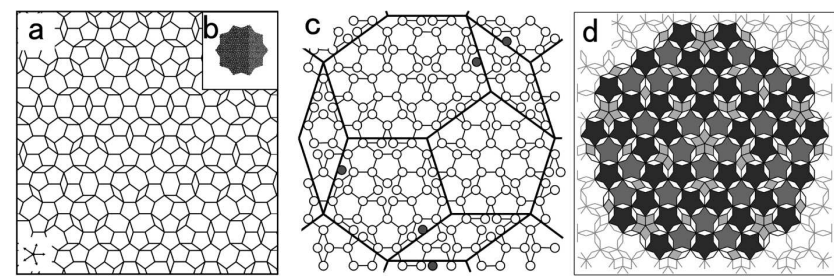

Fig. 4. (a) Ideal pentagonal tiling generated by the window (b) in perpendicular space, (c) TM atoms on the A plane, placed in the ideal pentagonal tiling of (a), and (d); occupation domains of the TM atom positions of (c). Black circles in (c) are extra TM atoms appearing from decagonal symmetry of the occupation domains of (d). Black and gray star-shaped domains in (d) are produced from TM atoms in pentagonal frames with opposite orientations respectively and light gray fat rhombuses and white thin rhombuses result from TM atoms in thin rhombic and squashed hexagonalframes in (c), respectively.
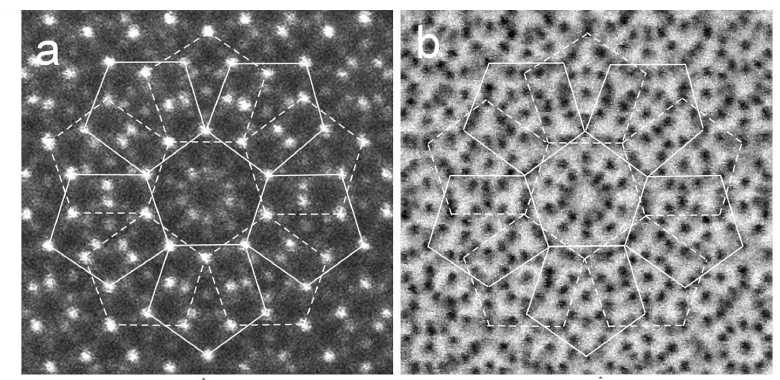

$$
\text { C }
$$

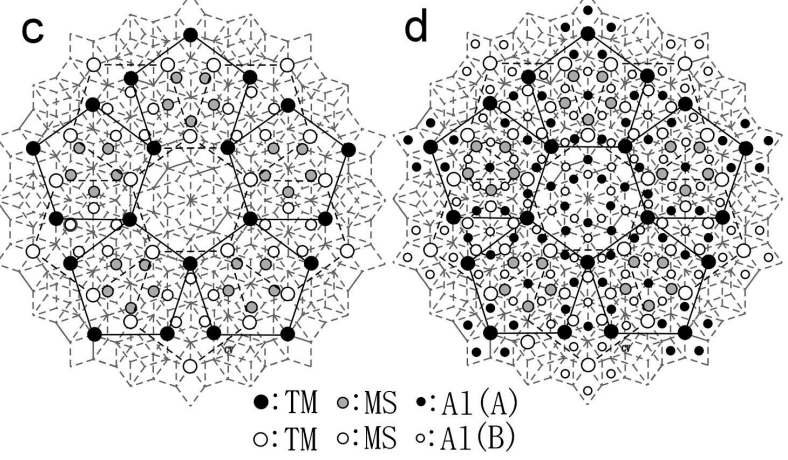

Fig. 5. HAADF-STEM (a) and ABF-STEM (b) images, an arrangement of TM and MSs (c), and a structure model (d) including $\mathrm{Al}$ atoms for the $3.2 \mathrm{~nm}$ cluster. Note that inside five bright dots in the double pentagonal arrangements in the HAADF-STEM image of (a) are MSs and outside five dots correspond to TM atoms and/or MSs, and that the ring contrasts with ten dark dots in (b) correspond to the projection of five MSs and five $\mathrm{Al}$ atoms, which are located on different planes. Backgrounds show pentagonal lattices of an edge-length of $0.25 \mathrm{~nm}$.

is generated by the projection of a five-dimensional (5D) hyper-cubic lattice through a window of Fig. $4 \mathrm{~b}$, as shown in Fig. 4c. All the TM atoms in the ideal pentagonal tiling are denoted by 5D hyper-cubic indices and are projected on the occupation domains with various forms in perpendicular space. In the occupation domains of
Fig. 4d, black and gray star-shaped domains are produced by TM atoms in pentagonal frames with opposite orientations and the light gray fat rhombuses as well as the white thin rhombuses result from TM atoms in the thin rhombic and squashed hexagonal frames of Fig. 4c respectively.

The TM and MSs atomic columns are located at the vertices of a Penrose tiling with an edge-length of $0.25 \mathrm{~nm}$, but $\mathrm{Al}$ atomic columns are considered to be shifted from the vertices based on the structures of the crystalline approximants of $\mathrm{W}-(\mathrm{AlCoNi})[10]$ and $\tau^{2}$ $\mathrm{Al}_{3} \mathrm{Co}$ [2]. Also, it is hard to determine the exact positions of $\mathrm{Al}$ atoms from the ABF image. However, it is worthwhile to discuss the structure of the $3.2 \mathrm{~nm}$ cluster from the observed HAADF- and ABF-STEM images. Figure 5 shows the observed HAADF- and ABF-STEM images and the structure model of the $3.2 \mathrm{~nm}$ cluster. The arrangement of TM atoms and MSs in Fig. 5c was directly derived from the HAADF-image of Fig. 5a, and the structure Fig. 5d was obtained from the ABF-STEM image of Fig. 5b, assuming that all $\mathrm{Al}$ atoms are located at the vertices of a Penrose tiling with an edge-length of $0.25 \mathrm{~nm}$. In the arrangement of TM atoms and MSs in Fig. 5c, one can see that the innermost five bright dots in the double pentagonal arrangements in the HAADFSTEM image of Fig. 5a are MSs and outer five dots correspond to TM atoms and/or MSs. As can be seen in Fig. 5d, the ring contrasts formed by ten dark dots in the ABF-STEM image of Fig. 5b are produced by the projection of five MSs and five $\mathrm{Al}$ atoms, which are located on different planes, as indicated by large circles in Fig. 5d. The TM atoms, Al atoms, and MSs in Fig. 5d are represented as dark dots in the ABF-STEM image of Fig. 5b. It should be mentioned here that the structure model of the $3.2 \mathrm{~nm}$ cluster in Fig. $5 \mathrm{~d}$ is similar to that proposed in the previous paper [9], except for the existence of MSs.

\section{Summary}

The structure of an $\mathrm{Al}-\mathrm{Fe}-\mathrm{Ni}$ decagonal quasicrystal with two quasiperiodic planes along the periodic axis was examined by Cs-corrected HAADF- and ABF-STEM. The arrangements of TM atoms and MSs of $\mathrm{Al}$ and TM atoms in the two quasiperiodic planes were determined from the arrangements of bright dots in the observed HAADF-STEM image. The TM atoms on the two quasiperiodic planes are arranged on a pentagonal tiling of an edge-length of $0.76 \mathrm{~nm}$, and pentagonal arrangements of MSs are located in pentagonal tiles with definite directions in the pentagonal tiling. The observed ABF- and HAADF-STEM images clearly show that large decagonal columnar clusters with $3.2 \mathrm{~nm}$ diameter are arranged on a pentagonal tiling of an edge-length of $3.2 \mathrm{~nm}$. The arrangement of $\mathrm{Al}$ atoms as well as TM atoms and MSs in the large decagonal atom cluster was interpreted from the observed HAADF- and ABF-STEM images.

The arrangements of TM atoms, which were determined from the observed HAADF-STEM image, are 
placed in an ideal pentagonal tiling with an edge-length of $3.2 \mathrm{~nm}$, which is generated by the projection of a fivedimensional hyper-cubic lattice. All the TM atoms in the ideal pentagonal tiling are denoted by 5 D hyper-cubic indices and are projected on the occupation domains with various forms in perpendicular space.

\section{References}

[1] A. Yasuhara, K. Saito, K. Hiraga, in: Proc. of Aperiodic 2012, Eds.: S. Schmid, R.L. Withers, R. Lifshitz, Springer, Berlin 2013, p. 219.

[2] K. Sugiyama, A. Yasuhara, K. Hiraga, in: Ref. [1], p. 237.

[3] K. Yubuta, A. Yasuhara, K. Hiraga, in: Ref. [1], p. 225 .
[4] K. Hiraga, A. Yasuhara, Mater. Trans. 54, 493 (2013).

[5] K. Hiraga, A. Yasuhara, Mater. Trans. 54, 720 (2013).

[6] A.P. Sai, A. Inoue, T. Masumoto, Mater. Trans. 30, 150 (1989).

[7] U. Lemmerz, B. Grushko, C. Freiburg, M. Jansen, Philos. Mag. Lett. 69, 141 (1994).

[8] K. Hiraga, K. Yubuta, K.-T. Prak, J. Mater. Res. 11, 1702 (1996).

[9] K. Hiraga, T. Ohsuna, Mater. Trans. 42, 894 (2001).

[10] K. Sugiyama, S. Nishimura, K. Hiraga, J. Alloys Comp. 342, 65 (2002). 